\title{
A Comprehensive Review of Viscosupplementation in Osteoarthritis of the Knee
}

Jacquelin Peck, MD', Annabel Slovek, AGACNP², Paulo Miro, BS ${ }^{3}$, Neeraj Vij, BS 3 , Blake Traube, BS ${ }^{3}$, Christopher Lee, $\mathrm{MD}^{4}{ }^{\mathrm{a}}$, Amnon A. Berger, MD, Ph.D. ${ }^{5}$, Hisham Kassem, MD', Alan D. Kaye, MD, Ph.D. ${ }^{6}$, William F. Sherman, MD, MBA? Alaa Abd-Elsayed, MD, $\mathrm{MPH}^{8}$

1 Department of Anesthesiology, Mount Sinai Medical Center, Miami Beach, FL, ${ }^{2}$ Valley Anesthesiology and Pain Consultants - Envision Physician Services, Phoenix, ${ }^{3}$ Department of Anesthesiology, University of Arizona College of Medicine-Phoenix, AZ, ${ }^{4}$ Department of Internal Medicine, Creighton University School of Medicine-Phoenix Regional Campus, Phoenix, AZ, 5 Department of Anesthesia, Critical Care, and Pain Medicine, Beth Israel Deaconess Medical Center, Harvard Medical School, Boston, MA, ${ }^{6}$ Department of Anesthesiology, Louisiana State University Shreveport, LA, 7 Department of Orthopaedic Surgery, Tulane University, New Orleans, LA, 8 Department of Anesthesiology, University of Wisconsin School of Medicine and Public Health, Madison, WI

Keywords: chronic pain, injection therapy, osteoarthritis, degenerative joint disease, sodium hyaluronate, hyaluronic acid

https://doi.org/10.52965/001c.25549

\section{Orthopedic Reviews}

Vol. 13, Issue 1, 2021

\section{Purpose of Review}

The purpose of this systematic review is to discuss emerging evidence in the field of viscosupplementation for chronic knee pain secondary to Osteoarthritis (OA). This review focuses on types of viscosupplementation that are clinically available currently, evidence to support their use, contraindications, and adverse events.

\section{Recent Findings}

OA, also known as degenerative joint disease, is the most common form of arthritis in the United States, affecting 54.4 million, or $22.7 \%$ of the adult population. The knee is the most common joint affected in OA, with up to $41 \%$ involvement, $30 \%$ in the hands, and $19 \%$ in the hips. The pathophysiology of OA is complex, with contributing factors including mechanical stress to the joint, as well as many person-specific factors such as genetic susceptibility, ethnicity, nutrition, and sex. Treatment modalities include weight control, exercise, non-steroidal and steroidal anti-inflammatory drugs, opioids, intra-articular platelet-rich plasma, placebo, corticosteroid injection, intra-articular viscosupplementation, and surgery. Viscosupplementation consists of injection of hyaluronic acid (HA) into affected joints, intending to restore the physiologic viscoelasticity in the synovial fluid (SF) in the absence of inflammation. HA has also been shown to downregulate pro-inflammatory factors, such as PGE2 and NFkB, and proteases and proteinases known to break down the joint matrix.

The contraindications for HA injection are similar to any other injection therapy, and adverse events are usually mild, local, and transient. Viscosupplementation (VS) is effective over placebo and more effective than NSAIDs and corticosteroids in pain reduction and improved functionality; however, guidelines recommend neither for nor against its use, demonstrating variability in the existing evidence base. Current VS options divide primarily into native vs. cross-linked and low-molecular-weight vs. high-molecular-weight. Current treatment options include Hylan g-f-20, Sodium Hyaluronate preparations (Suparts Fx, Euflexxa, Gelsyn-3, Durolane, Hyalgen), single-use agents (Gel-One, Synvisc-One, Monovisc), and Hyaluronan (Orthovisc, Monovisc, Hymovic). They share a common safety profile, and all have evidence supporting their efficacy. Their specific details are reviewed here.

\footnotetext{
a Corresponding author:

Christopher Lee, MD

St. Joseph Hospital \& Medical Center

Department of Internal Medicine

500 West Thomas Rd

Phoenix, AZ 85013

Chrislee0621@gmail.com
} 


\begin{abstract}
Summary
$\mathrm{OA}$ is the most common form of arthritis. It is a chronic, debilitating illness with a high impact on the functionality and quality of life of a significant part of the population in the western world. Treatments include medical management, physical therapy, activity modification, injection, and surgery. VS effectively reduces pain, increases functionality, and delays surgery in the knee to treat osteoarthritis. While previous studies have demonstrated variable results, more evidence is becoming available generally supportive of the benefit of VS in the treatment of knee OA.
\end{abstract}

\section{INTRODUCTION}

\section{OSTEOARTHRITIS OF THE KNEE}

Osteoarthritis (OA), also known as degenerative joint disease, is the most common form of arthritis in the United States, affecting 54.4 million, or $22.7 \%$ of the adult population. ${ }^{1}$ Of those affected, studies have reported that 22.7 million suffered from arthritis-attributable activity limitations. $^{2}$ The economic burden associated with OA is significant and multifactorial, including costs related to treatment, hospitalizations, work-related losses, and home-care costs. ${ }^{3}$ The knee is the most common joint affected in OA, with up to $41 \%$ involvement, compared to $30 \%$ in the hands and $19 \%$ in the hips. ${ }^{4,5}$ Knee OA, specifically, has doubled in prevalence since the mid-20th century, and has been estimated to have a lifetime direct per-person costs for symptomatic patients of $\$ 12,400.6,7$ OA has also been shown to substantially decrease quality of life, with studies estimating 86 million quality-adjusted life-years lost due to knee OA alone. ${ }^{8}$

\section{TREATMENT METHODS}

The pathophysiology of OA is complex, with contributing factors including mechanical stress to the joint and many person-specific factors such as genetic susceptibility, ethnicity, nutrition, and sex. ${ }^{9,10}$ Consequently, the range of treatment options for the management of knee OA is also multifaceted. Treatment methods can be classified into three major divisions: non-pharmacological, pharmacological, and interventional. Treatment modalities include weight control, exercise, non-steroidal and steroidal antiinflammatory drugs, opioids, intra-articular platelet-rich plasma, placebo, or corticosteroid injection, intra-articular viscosupplementation, and surgery. ${ }^{11}$ Interventional treatments for OA are the most recent of these, with viscosupplementation introduced in the late 19th century.

\section{VISCOSUPPLEMENTATION}

Viscosupplementation consists of injection of HA into affected joints, intending to restore the physiologic viscoelasticity in the synovial fluid (SF) in the absence of inflammation. ${ }^{12}$ SF is a solution found in the cavity of synovial joints, with the primary role of minimizing friction between the surrounding articular cartilage during movement. Hyaluronic acid (HA) is a naturally occurring and integral part of healthy cartilage and SF. It is composed of repeating disaccharide units formed by $N$-acetyl-d-glucosamine and d-glucuronic acid. Bothner \& Wik et al. showed that the molecular composition and rheological properties of HA are fundamental to the proper lubrication of joints. ${ }^{13}$ In addition to its lubricant properties, HA has many chondroprotective biochemical functions. Wang et al. showed that HA down-regulated the expression of pro-inflammatory cytokines through its interaction with fibroblast-like synoviocytes in the joint space. ${ }^{14}$ Furthermore, Yasuda et al. showed that HA suppresses macrophage production of Prostaglandin E2 via downregulation of NF-kappaB. ${ }^{15}$ Lastly, Sasaki et al. showed that the expression and production of matrix metalloproteinases, proteases involved in the degradation of matrix proteins that contribute to the integrity of the joint, is decreased in the presence of HA. ${ }^{16}$ Many hyaluronic acid derivatives are currently being used in the treatment of knee osteoarthritis, whether be it cross-linked hyaluronic acid such as Hylan g-f 20, sodium hyaluronate (the salt form of hyaluronic acid, which has a smaller molecular weight but greater molecular stability), or hyaluronan (a high molecular weight polysaccharide found in hyaluronic acid).

\section{CONTRAINDICATIONS AND ADVERSE EVENTS}

Contraindications to HA include known hypersensitivity to HA products, pediatric patients, bacteremic patients, and those with active local infection in the articular target of injection. Adverse events associated with viscosupplementation are not well reported in the literature, and the ones reported have varied rates of incidence. Reported adverse events include pain at the site of injection, local skin reactions, pseudoseptic reactions, septic arthritis, and local joint pain and swelling. ${ }^{17}$

\section{EVIDENCE AND GUIDELINES}

A 2006 Cochrane Review, including seventy-six randomized controlled trials evaluating viscosupplementation with a diagnosis of knee OA in at least one branch, supported the efficacy of viscosupplementation against placebo. The authors report beneficial effects on pain, physical function, and global assessment, with the strongest findings between $5-13$ weeks post-injection. ${ }^{18}$ In some studies, viscosupplementation was similarly effective compared to NSAIDs, with more prolonged effects than corticosteroids. However, the authors caution that there was insufficient sample size to definitively compare classes of viscosupplements. In addition, they stated that limited evidence suggests that differences may exist in both safety and efficacy between the two classes.

Despite conclusions from the 2006 Cochrane Review, currently available guidelines and more recent meta-analyses and systematic reviews have conflicting recommendations. The latest guidelines published by the American 
Academy of Orthopaedic Surgeons (AAOS) in 2013 state that HA for patients with symptomatic OA of the knee cannot be recommended noting that most of the literature surrounding viscosupplementation has a significant degree of publication bias. ${ }^{19}$ The 2014 Osteoarthritis Research Society International (OARSI) guidelines offer an "uncertain” recommendation for intraarticular HA in knee OA. ${ }^{20}$ Lastly, the American College of Rheumatology (ACR), in their 2012 guidelines, states that they have no recommendations regarding the use of intraarticular hyaluronates. ${ }^{21}$ There have been several systematic reviews and meta-analyses with differing results as well. Rutjes et al. in 2012 concluded through a comprehensive systematic review and metaanalysis that viscosupplementation is associated with a clinically irrelevant benefit and an increased risk for adverse events. $^{22}$ In 2015, Richette and colleagues concluded through an updated meta-analysis of trials with low risk of bias that HA supplementation provided a moderate but real benefit for patients with knee OA. ${ }^{23}$ The following year, Xiao et al. led a PRISMA-compliant systematic review of 12 meta-analyses, concluding that HA is both an effective and safe intervention in treating knee OA. ${ }^{24}$ Published guidelines surrounding the use of viscosupplementation remain uncertain, and data from meta-analyses or systematic reviews have been inconsistent. Furthermore, a wide variety of hyaluronic acid derivatives are available and used in treating knee osteoarthritis, with little consensus on the clinical superiority of one agent over another.

\section{PURPOSE}

The purpose of this systematic review is to discuss emerging evidence in the field of viscosupplementation for chronic knee pain secondary to OA. We discuss systematic reviews, meta-analyses, and randomized controlled trials relating to agents used for viscosupplementation and published within the past 5 years.

\section{HYLAN G-F-20}

Many viscosupplementation products exist. This section will focus on Hylan g-f20 specifically. Two classifications primarily distinguish HA products from one another; native vs. cross-linked and low-molecular-weight vs. high molecular weight HA. Hylan g-f20 is a cross-linked product derived from rooster comb $\mathrm{HA}$, with a higher molecular weight $(6000 \mathrm{kDa}){ }^{25}$ Hylan g-f20 is marketed under the brand name Synvisc and Synvisc-One. Synvisc is administered in three separate doses ( $2 \mathrm{~mL}, 16 \mathrm{mg}$ each), while Synvisc-one is administered once $(6 \mathrm{~mL}, 48 \mathrm{mg}){ }^{26}$ The difference is solely administration convenience, with no trial showing a difference in efficacy between the two. Hylan gf-20 mimics naturally occurring HA reducing nociception, promoting synthesis of extracellular matrix proteins, and suppressing inflammatory mediators. ${ }^{27}$ However, its unique chemical properties may provide additional benefits. Wobig et al. concluded that the higher molecular weight of Hylan g-f20 makes this product more elastoviscous compared to other HA preparations with lower molecular weights. ${ }^{28}$ Wobig also suggested that the pain-relieving effect reported in patients who receive viscosupplementation is directly re- lated to the elastoviscosity of the product used for intra-articular injection. ${ }^{28}$ Cross-linking of the formulation is also intended to allow for longer-lasting efficacy by increasing resistance to degradation within the knee joint.

\section{SAFETY PROFILE}

Existing literature does not attribute any unique adverse events to the use of Hylan g-f20 specifically. Two large studies suggest that the rate of mild adverse reactions does not increase with the use of Hylan g-f20. In a recent metaanalysis, Zhao et al. compare Hylan g-f20 to lower molecular weight HAs and conclude that there is no evidence of increased risk of treatment-related mild adverse events with Hylan g-f20 injections. ${ }^{29}$ In a multicenter longitudinal study conducted in 2015, Yan et al. report the adverse event profile of Hylan- $\mathrm{g}$-f20 to be mild and self-limiting. The majority of events are local inflammatory reactions. ${ }^{30}$ Following a randomized controlled trial, Leopold et al. reported that local reactions to Hylan g-f20 occurred more often in those receiving more than a single injection, raising concern for multiple-course treatment with Hylan g-f20.31 Lastly, Goldberg and colleagues suggested that there may be a higher incidence of pseudoseptic reactions with the use of Hylan g-f20 compared to other HA preparations. ${ }^{32}$ They hypothesize that Hylan g-f20 may have increased immunogenicity due to the chemical cross-linking of the molecule, as well as possible contamination from chicken proteins and other components of rooster combs during the cross-linking process. ${ }^{32}$ This hypothesis was supported by Ishikawa and colleagues, who concluded that Hylan g-f20 was less biocompatible and had higher immunogenicity compared to a different cross-linked HA formulation, with correspondingly higher rates of pseudoseptic reactions. ${ }^{33}$

\section{EFFICACY}

There have been several large, high-quality studies in the last five years investigating the effectiveness of Hylan g-f20. Waddell et al. report that, from a large cohort of 1,863 patients with grade IV OA of the knee, $75 \%$ of patients receiving Hylan g-f20 injections delayed the need for total knee replacement by 7 years or more. ${ }^{34}$ However, this study evaluated Hylan g-f20 alone and did not compare it to other traditional treatments for OA or placebo. The authors also found a slight increase in knee synovitis with repeat injection, though the cases were generally mild or moderate in severity. Similarly, Kearey et al. concluded that singleinjection Hylan G-f20 led to clinically significant improvements in pain and function, with results most pronounced at the 3 -month and 6-month period. ${ }^{35}$ However, the authors suggest that the symptomatic relief provided by Hylan g-f20 may be reduced as the patient's age increases. Tammachote et al. concluded that a single injection of Hylan g-f20 provided similar symptomatic relief at the 6 months compared to a single injection of corticosteroids, with the corticosteroid injection resulting in better pain control and functional improvement in the early weeks following treatment. ${ }^{36}$ More recently, Dai and colleagues published a meta-analysis finding no clinically important difference in outcomes, including pain relief and functional improve- 
ment between Hylan and HA injections. ${ }^{37}$ Furthermore, they discouraged the use of Hylan over HA due to its higher costs. However, a similar study by Migliore and colleagues indicated that Hylan g-f20 is a cost-effective treatment, even when compared to NSAIDs. ${ }^{38}$ This suggests that Hylan g-f20 may be cost-effective when compared to pharmacologic treatment but cost-ineffective when compared to other viscosupplementation formulations

\section{SUMMARY}

In summary, the data suggests that Hylan g-f20 may be an effective interventional treatment for knee OA and may delay time for total knee replacement surgery. The clinical benefit of Hylan g-f20 seems to be most clinically noticeable two to nine months following the initiation of treatment. Data comparing clinical benefits to other agents is conflicting, heterogeneous, and difficult to conclude due to studies using different thresholds for determining a clinically significant difference. In terms of safety and adverse events, Hylan g-f20 is not associated with a greater incidence of mild adverse events. Still, it may be associated with a greater incidence of pseudoseptic reactions, especially in those receiving multiple injections. The suspected increased immunogenicity associated with Hylan g-f20 may render it an inferior option for long-term intra-articular treatment. Hylan g-f20 may be a suitable option in the elderly population who cannot tolerate pharmacologic treatment but may also experience decreased symptom relief as the patient population age increases.

\section{SODIUM HYALURONATE}

\section{BACKGROUND}

Sodium hyaluronate is a salt form of hyaluronic acid and is a glycosaminoglycan formed from long-chain disaccharide $\mathrm{Na}$-glucuronate-N-acetylglucosamine units. It is a naturally occurring substance found within connective tissues. When used as a viscosupplement in treating knee osteoarthritis, it improves symptomatology by acting as a lubricant and shock absorber. Still, it has also shown benefit surpassing its duration of action, which has been linked to anti-inflammatory properties. ${ }^{39}$ Preparations include Supports, Euflexxa, Gelsyn, Durolane, and Hyalgan. These are typically used once the first medical management has failed but may delay the need for knee replacement surgery. ${ }^{39}$

\section{SUPARTS FX}

SUPARTZ FX is an injectable solution containing sodium hyaluronate, a conjugated salt form of hyaluronic acid. Supartz Fx is derived from chicken combs extract and is given weekly injections of $25 \mathrm{mg}$ for 3-5 weeks. It has been shown to have a more significant benefit for both pain and function when compared with other non-surgical treatments. ${ }^{40}$

\section{EUFLEXXA}

Euflexxa contains sodium hyaluronate developed using bacterial fermentation. It is given as weekly injections of 20 $\mathrm{mg}$, for a total of three injections. Like Supartz Fx, it is not cross-linked and has an intermediate molecular weight of 24,000-3,600 kDa. Euflexxa has been associated with adverse effects including joint pain, back pain, limb pain, muscle pain, and joint swelling. No literature evaluates the use of Euflexxa in pregnant women or children less than 18 years of age. However, it has been deemed safe and is FDA approved for the remainder of the general population. ${ }^{41}$

\section{GELSYN-3}

Gelsyn-3 (Sinovial ${ }^{\circledR}$ ) is non-crosslinked $0.84 \%$ sodium hyaluronate of bio-fermentative origin and is administered in a three-injection seriGelsyn, Durolane, es. As with any injection, risks include general knee pain, warmth, and redness at the injection site. ${ }^{42}$ In a randomized controlled trial evaluating Gelsyn-3 compared to Hylan G-F20, both preparations worked similarly well with patients experiencing approximately 32.5 point reductions in Western Ontario and McMaster Universities (WOMAC) pain subscores at 26 weeks. Both viscosupplements reduced joint pain and stiffness for up to 6 months, improved quality of life, and delayed the need for total knee replacement surgery. ${ }^{43}$

\section{DUROLANE}

Durolane is a single-injection product using a cross-linked sodium hyaluronate agent derived from bio-fermentation, with a half-life of approximately four weeks. In a 2018 meta-analysis, Leighton et al. found that Durolane provides significant improvements in pain, physical function, and quality of life for knee osteoarthritis. They also found Durolane to be superior or non-inferior to treatments including placebo, corticosteroids, Hylan G-F20, and multiinjection sodium hyaluronate. Leighton et al. found that 23 of the 175 patients receiving Durolane experienced adverse effects including post-injection pain, joint swelling, and pseudoseptic reaction; however, these were considered mild and transient. ${ }^{44}$

\section{HYALGEN}

Hyalgen is a high molecular weight hyaluronic acid product (500,000-730,000 daltons) derived from purified rooster comb extract. Because it is not cross-linked, it is given in $20 \mathrm{mg}$ doses over a 3 -injection course. It has provided longlasting pain relief for up to 6 months and is safe for repeat use up to 30 months. US clinical trials have shown adverse effects including pain at the injection site, gastrointestinal discomfort, headaches, local ecchymosis, and pruritus. Because it is a purified fraction of sodium hyaluronate extracted from roosters, allergic and anaphylactic are slightly more common than with many other HA viscosupplements. Though these adverse effects remain rare, physicians should exercise caution in the use of this product with patients with known allergic reactions to HA products. ${ }^{40,45}$

\section{ADDITIONAL REVIEW ARTICLES, META-ANALYSES, AND RANDOMIZED CONTROLLED TRIALS}

Emerging research has demonstrated the clinical efficacy of sodium hyaluronate in the treatment of knee osteoarthritis. A 2017 retrospective questionnaire-based survey con- 
ducted by Miller et al. found that a multimodal treatment approach-five weekly injections of Hyalgen, structured physical therapy, knee bracing, and patient education-resulted in a $60 \%$ reduction in WOMAC pain subscores and had an incremental cost-effectiveness ratio (ICER) of 6,000 per quality-adjusted life-year (QALY). ${ }^{46}$ However, nuances in viscosupplement agent selection require additional investigation.

Supartz Fx has been on the US market since 2001 and it is well established as an effective method for reducing pain and improving clinical outcomes for patients with osteoarthritis (OA.) Bannuru et al. 2016 sought to determine the safety of repeat courses of SUPARTZ injections. This systematic review found SUPARTZ repeat injections to be safe with an overall adverse event rate of 0.008 ( $95 \%$ confidence interval of 0.001-0.055.), which supports repeat SUPARTZ injections in the symptomatic management of osteoarthritis in the knee. ${ }^{39,40}$ Curran et al. 2010 found no significant difference in the incidence of adverse effects between Supartz and control groups using phosphatebuffered saline. ${ }^{47}$ Further, data exists that suggests SUPARTZ may be beneficial in improving muscle strength, gait pattern, and balance though further research is still needed to confirm these findings. ${ }^{39}$

Single-use viscosupplementation agents are also available and attract growing consideration because of increased cost-effectiveness and patient convenience with decreased exposure to risks associated with knee injection, including injection pain, patient discomfort, injection-related inflammation, and erythema. Single-injection HA products currently available include Gel-One, Synvisc-One, and Monovisc. ${ }^{40}$ Conflicting evidence exists regarding the relative effectiveness of these agents. Al-Omran et al. showed 3 injections of Synvisc to be statistically significantly more effective in improving than one $60 \mathrm{mg} / 3 \mathrm{~mL}$ injection of Durolane in an initial double-blind, randomized study. Further, Diracoglu et al. 2016 showed Monovisc (a single dose injection) to have no statistical differences compared to Adavant (3 standard linear injections) regarding VAS-pain and WOMAC scores after the injections, but found single injection Monovisc to be significantly inferior at 6 months regarding VAS-pain scores. ${ }^{48}$ However, in a meta-analysis of single-injection products, Vincent et al. found mono-injections to produce results similar to multi-injections. ${ }^{49} \mathrm{Fi}$ nally, in a 2017 meta-analysis including twenty-four studies evaluating weekly Hyalgan knee intraarticular injections over three vs. five weeks in 2168 cumulative study participants, Stitek et al. found no statistical difference between the 3 and 5-week courses. This may support HA administration over fewer injections without diminishing clinical benefits. Given these varying results, further systematic analyses are needed to explore the use of single-injection HA products. 50

\section{SUMMARY}

Intraarticular sodium hyaluronate injections appear to be a safe and effective treatment for symptomatic treatment of $\mathrm{OA}$. These agents provide a cost-effective alternative therapy for patients who have been unsuccessful with other non-operative methods and have been deemed as safe and effective among healthy adults. However, reported adverse effects include arthralgia, arthropathy/arthrosis/arthritis, back pain, injection-site reaction/pain, and headache. ${ }^{47}$ These reactions are generally mild to moderate in severity. ${ }^{39,40}$ The comparative efficacy of single-injection HA products has mixed results in the literature and requires further investigation. ${ }^{48-51}$

\section{HYALURONAN}

\section{BACKGROUND}

Hyaluronan (HA) is a high-molecular-weight extracellular matrix (ECM) polysaccharide and hyaluronic acid derivative, particularly in soft connective tissues. ${ }^{52}$ It has various functions in development, growth, and tissue regeneration. Similar to Hyaluronic Acid, Hyaluronan is a polymer used as a viscosupplement that may directly modulate the expression of inflammatory genes via cell surface receptors - such as CD4, RHAMM, and Toll-like receptors - and regulation of downstream signaling. ${ }^{52,53}$ Following synthesis via HA synthases as a protein-free polysaccharide at the cell membrane, it may associate with receptors or diffuse across the membrane into the ECM, contributing to various biological effects as part of complexes with specific binding proteins. HA may also be cross-linked to form matrices. ${ }^{52}$ These various macromolecular matrixes are implicated in the regulation of inflammatory cell migration and adhesion, acting as an influence on anti-inflammatory and pro-inflammatory activity. Aside from the potential roles of HA in inflammation pathways, the polymer acts as a viscoelastic shock absorber by virtue of its size, its presence in the ECM, and its rheological properties. ${ }^{52,53}$

Hyaluronan's unique function as a viscosupplementation agent is traditionally attributed mainly to its viscoelastic properties related to a high molecular weight and gel formulation. The viscoelastic properties attributed to hyaluronan contributed to its initial use as a viscosupplement. However, the additional benefit has been attributed to potential inflammatory and biological pathway enhancements. ${ }^{52,53}$ It is administered in treating knee osteoarthritis as an injection directly into the joint, where viscoelastic properties work to reduce the mechanical forces and inflammatory pathways driving the pathogenesis and symptomatic presentation of arthritis. ${ }^{52}$ Machado et al. recently suggested that the clinical response to HA may depend on the intrinsic properties of the polysaccharide, whether hyaluronan or another similar polysaccharide, rather than on the rheology. ${ }^{53}$ The mechanism of action behind viscosupplements is multifactorial and remains under evaluation.

Risks associated with hyaluronan are mild and similar to those of other viscosupplements, including allergies, injection site pain and inflammation, infection, and potential temporary arthritic flares. Other rare side effects include itching, numbness/tingling, headache, dizziness, and back pain. 52,53

Multiple brand-name formulations of hyaluronan are used for intraarticular injection in the management of knee osteoarthritis. These include Orthovisc, Monovisc, and Hymovis. Several differences exist between formulations. Or- 
thovisc hyaluronan particles are derived from rooster combs, while Hymovis and Monovisc hyaluronan are extracted and purified from bacterial fermentation, free from any animal proteins. Monovisc is administered as a single injection, while Hymovis is administered in 2 injections, and Orthovisc is administered in 3-4 injections spaced one week apart. Monovisc is considered moderate molecular weight and is cross-linked at time of injection, Orthovisc is deemed to be moderate molecular weight and is not crosslinked at time of injection, and Hymovis is considered low molecular weight and is not cross-linked at time of injection. ${ }^{52}$

\section{SYSTEMATIC REVIEWS AND META-ANALYSES}

Multiple systematic reviews and meta-analyses have been published recently about hyaluronan injections. In examining the use of intra-articular hyaluronan therapy vs. placebo, Richardson et al. ultimately concluded that the numerous randomized controlled trials (RCTs) and metaanalyses demonstrated clinical benefit of hyaluronan generally that the active ingredient HA itself did not necessarily cause such benefit. ${ }^{52}$ The reviewers maintained that much of the benefit was attributable to a placebo effect. The small benefit of hyaluronan over placebo found in some of the examined studies was unknown clinical significance. The authors reported that hyaluronan was comparable to injectable corticosteroids for long-term relief, though less effective than injectable corticosteroids for more short-term benefit, and concluded that it may be an appropriate and effective therapy. 52

Another meta-analysis by Yanhong et al. compared platelet-rich plasma (PRP) to hyaluronan injections for knee osteoarthritis. This study included fifteen RCTs and concluded that PRP injections reduced pain more effectively than hyaluronan at both 6-month and 12-month follow-up periods and provided more functional improvement in individuals. They also found no significant difference in adverse effects in PRP vs. HA. ${ }^{54}$ A meta-analysis by Qingsong et al. compared hyaluronan with intraarticular oxygen-ozone, concluding that intraarticular hyaluronan was associated with a significant reduction in visual analog scale-scored pain relative to oxygen-ozone. ${ }^{55}$ A systematic review by Gregori et al. looked much more broadly at 47 different RCTs that, as a collective, covered 31 different pharmacologic interventions (including but not limited to analgesics, intra-articular injections, and bone-acting agents) for long-term pain control of osteoarthritis. While this review eventually affirmed uncertainty between all pharmacologic treatments and placebo in long-term control specifically, the authors concluded that hyaluronan showed no association with long-term pain improvement. ${ }^{56}$

Two different reviews and meta-analyses compared hyaluronan products with multiple injections (e.g., 2 or more weekly injections) vs. monoinjections (e.g., one-time injection) for knee osteoarthritis. Concoff et al. established that hyaluronan administration with $2-4$ injections or $\geqslant 5$ injections provided significant pain relief over intraarticular saline, while monoinjection courses did not. ${ }^{57} 2-4$ week injection courses were found to be most effective in pain control. ${ }^{57}$ Vincent et al., however, determined that monoin- jections produce similar results to multiple injections of hyaluronan with regard to pain relief from knee osteoarthritis. ${ }^{49}$

Finally, in a meta-analysis including six RCTs involving 310 cumulative patients, Dongjun et al. evaluated the use of hyaluronan following knee arthroscopy. They concluded that while hyaluronan significantly improved WOMAC Osteoarthritis Index scores, there was no substantial improvement in pain scores at 2,6 , or 12 weeks post-arthroscopy. ${ }^{58}$

\section{ADDITIONAL RANDOMIZED CONTROLLED TRIALS}

Additional randomized controlled studies on hyaluronan have also been recently completed and were not considered in the previously discussed reviews and meta-analyses. Henrotin et al. sought to further clarify and identify the biological and clinical biomarkers for the efficacy of Hymovis injections. They found that Hymovis significantly increased type II collagen turnover and overall collagen volume at injected, leaving this as a foundation for further understanding Hymovis's activity and efficacy. ${ }^{59}$ Hermans et al. concluded that intra-articular high molecular weight hyaluronan added to usual care is effective for treating knee osteoarthritis in working-age individuals, and Petterson et al. also found that Monovisc was safe and effective at providing clinically significant pain improvement within 2 weeks. ${ }^{60,61}$

Other recent trials have compared hyaluronan treatment to alternatives and supplements. Yoon et al. concluded that combining hyaluronan with polydeoxyribonucleotide (another injectable therapy for knee osteoarthritis) can also be considered for knee osteoarthritis treatment. However, further studies are needed to evaluate clinical benefits. ${ }^{62}$ Wang et al. established that patients receiving co-treatment with hyaluronan and corticosteroids experienced pain relief and improved knee function faster than either agent alone. However, there was no significant difference at 6 months. 63 Huang et al. also found that intra-articular PRP injections were comparable to corticosteroids and hyaluronan in pain improvement at 3-month follow-up, but PRP injections were superior in long-term pain relief. ${ }^{64}$

\section{SUMMARY}

Evidence surrounding the use of hyaluronan is inconclusive and contradictory. While many RCTs determine there is significant pain improvement with hyaluronan injections, similar success is not found in other RCTs, and larger-scale systematic reviews and meta-analyses of these studies have concluded that HA may not be considerably effective beyond the placebo effect. ${ }^{52,53}$ Many studies evaluating hyaluronan have been conducted on patients in more advanced age, though there is the promise that HA can provide clinical benefit in working-age individuals as well. ${ }^{60,61}$ Therapy appears to be most effective when delivered in 2-4 weekly injections, though further research is needed here as well. ${ }^{49,57}$ Treatment is typically safe with very few and rare adverse effects. Hyaluronan can be expensive, with Hymovis being the cheapest option at $\$ 872.00$ per dose from the average wholesaler. More affordable treatment options may also be considered before hyaluronan, as the cost may prove 
a limitation to some patients. Ultimately, evidence exists to support hyaluronan as a safe and effective option. Still, further comparison between hyaluronan and other viscosupplements should be completed to clarify each agent's role and relative efficacy.

\section{CONCLUSION}

OA is the most common form of arthritis in the United States, and knee OA is the most common form of OA. It is a prevalent disease in the Western World, causing significant pain and suffering and a functional limitation to a large part of the population. It carries tremendous costs in treatment, hospitalization, and productivity loss, the latter estimated at up to 86 million QALY.

Treatment of knee OA begins with conservative treatment through injection therapy and almost invariably culminates in surgery. Before surgery, the treatment options are aimed at reduction of pain, increased functional status, and delaying of surgery.

Viscosupplementation is part of the array of injection therapy available for OA. Current options include native and cross-linked, low-molecular or high-molecular-weight options. Here we reviewed the evidence supporting the use of more recent options, Hylan G-F 20, sodium hyaluronate preparations, and hyaluronan. These options are all relatively safe and side-effects are usually self-limited and local.

Though current guidelines are still inconclusive about using VS for knee OA, citing biased and low-quality evidence, there is an increasing body of evidence supporting VS over placebo and other types of injection therapy. Numerous meta-analyses and systematic reviews found a cumulative benefit in all of the goals mentioned earlier after considering bias. As more data becomes available, guidelines may likely change.

More research is required into upcoming single injection solutions, as these may make this therapy even more accessible and affordable. Preliminary evidence also supports the safety and benefit of multiple injections; however, more research should be performed into that aspect.

The evidence reviewed can give readers a comprehensive understanding of the use of VS for pain alleviation in knee OA.

\section{DISCLOSURES}

The following manuscript has not been previously published. No contributing author has a conflict of interest to declare.

\section{REFERENCES}

- OF IMPORTANCE: 19, 23, 28, 39

19. The current guidelines from the American Academy of Orthopaedic Surgeons for treatment of knee OA. The AAOS does not currently recommend VS for knee OA, citing biased evidence.

23. A meta-analysis and systematic review of VS with HA in knee OA reviewing only trials comparing with placebo and meeting criteria for low bias, finding a significant (though possibly small) advantage for VS in OA.

28. An RCT comparing Hylan G-F 20 to low-molecularweight HA, demonstrating superiority of Hylan G-F 20.

39. A review of available evidence to support the efficacy and safety of sodium hyaluronate (SUPARTZ) in knee OA.

\section{- OF MAJOR IMPORTANCE: $11,18,40$}

11. A systematic review covers the background and pathophysiology of knee OA and provides an overview of nonsurgical therapeutic options.

18. A Cochrane systematic review of viscosupplementation for OA of the knee from 2006 (first available) reviewing RCTs and concluding that VS effectively reduces pain and increases functionality in knee OA.

40. A systematic review and meta-analysis on the safety of repeated sodium hyaluronate (specifically SUPARTZ) injections in knee OA supporting the safety of repeat injections.

Submitted: June 30, 2021 EST, Accepted: July 03, 2021 EST 


\section{REFERENCES}

1. Barbour KE, Helmick CG, Boring M, Brady TJ. Vital Signs: Prevalence of Doctor-Diagnosed Arthritis and Arthritis-Attributable Activity Limitation - United States, 2013-2015. MMWR Morb Mortal Wkly Rep. 2017;66(9):246-253. doi:10.15585/mmwr.mm6609e1

2. Hootman JM, Helmick CG, Barbour KE, Theis KA, Boring MA. Updated Projected Prevalence of SelfReported Doctor-Diagnosed Arthritis and ArthritisAttributable Activity Limitation Among US Adults, 2015-2040. Arthritis Rheumatol (Hoboken, NJ). 2016;68(7):1582-1587. doi:10.1002/art.39692

3. Bitton R. The Economic Burden of Osteoarthritis. Am J Manag Care. 2009;15(8):230-235. doi:10.1002/ar $\underline{\mathrm{t} .1780290311}$

4. Felson DT, Zhang Y. An update on the epidemiology of knee and hip osteoarthritis with a view to prevention. Arthritis Rheum. 1998;41(8):1343-1355. doi:10.1002/1529-0131(19980 8) $41: 8$

5. Zhang Y, Jordan JM. Epidemiology of osteoarthritis. Clin Geriatr Med. 2010;26(3):355-369. doi:10.1016/j.cg er.2010.03.001

6. Wallace IJ, Worthington S, Felson DT, et al. Knee osteoarthritis has doubled in prevalence since the mid-20th century. Proc Natl Acad Sci USA. 2017;114(35):9332-9336. doi:10.1073/pnas.17038561 $\underline{14}$

7. Losina E, Paltiel AD, Weinstein AM, et al. Lifetime medical costs of knee osteoarthritis management in the United States: impact of extending indications for total knee arthroplasty. Arthritis Care Res (Hoboken). 2015;67(2):203-215. doi:10.1002/acr.22412

8. Losina E, Walensky RP, Reichmann WM, et al. Impact of obesity and knee osteoarthritis on morbidity and mortality in older Americans. Ann Intern Med. 2011;154(4):217-226. doi:10.7326/0003-4 819-154-4-201102150-00001

9. Neogi T, Zhang Y. Epidemiology of OA. Rheum Dis Clin North Am. 2013;39(1):1-19. doi:10.1016/j.rdc.201 $\underline{2.10 .004}$

10. Iannone F, Lapadula G. The pathophysiology of osteoarthritis. Aging Clin Exp Res. 2003;15(5):364-372.

11. Mora JC, Przkora R, Cruz-Almeida Y. Knee osteoarthritis: Pathophysiology and current treatment modalities. J Pain Res. 2018;11:2189-2196. doi:10.2147/JPR.S154002
12. de Rezende MU, de Campos GC.

Viscosupplementation. Rev Bras Ortop (English Ed). 2012;47(2):160-164. doi:10.1016/S2255-4971(15)3008 $\underline{0-X}$

13. Bothner H, Wik O. Rheology of hyaluronate. Acta Otolaryngol. 1987;104(S442):25-30. doi:10.3109/0001 $\underline{6488709102834}$

14. Wang CT, Lin YT, Chiang BL, Lin YH, Hou SM. High molecular weight hyaluronic acid downregulates the gene expression of osteoarthritisassociated cytokines and enzymes in fibroblast-like synoviocytes from patients with early osteoarthritis. Osteoarthr Cartil. 2006;14(12):1237-1247. doi:10.101 6/j.joca.2006.05.009

15. Yasuda T. Hyaluronan inhibits prostaglandin E2 production via CD44 in U937 human macrophages. Tohoku J Exp Med. 2010;220(3):229-235. doi:10.1620/t jem.220.229

16. Sasaki A, Sasaki K, Konttinen YT, et al. Hyaluronate inhibits the interleukin-1beta-induced expression of matrix metalloproteinase (MMP)-1 and MMP-3 in human synovial cells. Tohoku J Exp Med. 2004;204(2):99-107. doi:10.1620/tjem.204.99

17. Eisenberg Center at Oregon Health \& Science University. Three Treatments for Osteoarthritis of the Knee: Evidence Shows Lack of Benefit.; 2007.

18. Bellamy N, Campbell J, Robinson V, Gee T, Bourne $\mathrm{R}$, Wells G. Viscosupplementation for the treatment of osteoarthritis of the knee. Cochrane Database Syst Rev. 2006;(2):CD005321. doi:10.1002/14651858.CD00 5321.pub2

19. McGrory B, Weber K, Lynott JA, et al. The American Academy of Orthopaedic Surgeons Evidence-Based Clinical Practice Guideline on Surgical Management of Osteoarthritis of the Knee. J Bone Joint Surg Am. 2016;98(8):688-692. doi:10.2106/I BJS.15.01311

20. Mcalindon TE, Bannuru RR, Sullivan MC, et al. OARSI guidelines for the non-surgical management of knee osteoarthritis. Published online 2014. doi:1 0.1016/i.joca.2014.01.003

21. Hochberg MC, Altman RD, April KT, et al. American College of Rheumatology 2012 Recommendations for the Use of Nonpharmacologic and Pharmacologic Therapies in Osteoarthritis of the Hand, Hip, and Knee. Published online 2012. doi:10.1 002/acr.21596 
22. Rutjes AWS, Jüni $P$, da Costa BR, Trelle S, Nüesch E, Reichenbach S. Viscosupplementation for osteoarthritis of the knee: a systematic review and meta-analysis. Ann Intern Med. 2012;157(3):180-191. doi:10.7326/0003-4819-157-3-201208070-00473

23. Richette P, Chevalier X, Ea HK, et al. Hyaluronan for knee osteoarthritis: an updated meta-analysis of trials with low risk of bias. RMD Open.

2015;1(1):e000071. doi:10.1136/rmdopen-2015-00007 1

24. Xing D, Wang B, Liu Q, et al. Intra-articular Hyaluronic Acid in Treating Knee Osteoarthritis: A PRISMA-Compliant Systematic Review of Overlapping Meta-analysis. Sci Rep. 2016;6. doi:10.10 38/srep32790

25. Intra-articular viscosupplementation with hylan g-f 20 to treat osteoarthritis of the knee: an evidencebased analysis. Ont Health Technol Assess Ser. 2005;5(10):1-66.

26. Synvisc-One ${ }^{\circledR}$. Frequestly Asked Questions. Synvisc-One ${ }^{\circledR}$ Official Site. https://www.synviscone.c om/what-is-synvisc-one/synvisc-faqs

27. Moreland LW. Intra-articular hyaluronan (hyaluronic acid) and hylans for the treatment of osteoarthritis: mechanisms of action. Arthritis Res Ther. 2003;5(2):54. doi:10.1186/ar623

28. Wobig M, Bach G, Beks P, et al. The role of elastoviscosity in the efficacy of viscosupplementation for osteoarthritis of the knee: A comparison of hylan G-F 20 and a lowermolecular-weight hyaluronan. Clin Ther. 1999;21(9):1549-1562. doi:10.1016/S0149-2918(00)80 010-7

29. Zhao H, Liu H, Liang X, Li Y, Wang J, Liu C. Hylan G-F 20 Versus Low Molecular Weight Hyaluronic Acids for Knee Osteoarthritis: A Meta-Analysis. BioDrugs. 2016;30(5):387-396. doi:10.1007/s40259-01 6-0186-1

30. Yan $\mathrm{CH}$, Chan WL, Yuen WH, et al. Efficacy and safety of hylan G-F 20 injection in treatment of knee osteoarthritis in Chinese patients: Results of a prospective, multicentre, longitudinal study. Hong Kong Med J. 2015;21(4):327-332. doi:10.12809/hkmj14 $\underline{4329}$

31. Leopold SS, Warme WJ, Pettis PD, Shott S. Increased frequency of acute local reaction to intraarticular hylan GF-20 (synvisc) in patients receiving more than one course of treatment. J Bone Joint Surg Am. 2002;84(9):1619-1623. doi:10.2106/00004623-20 0209000-00015
32. Goldberg VM, Coutts RD. Pseudoseptic reactions to hylan viscosupplementation: diagnosis and treatment. Clin Orthop Relat Res. 2004;(419):130-137. doi:10.1097/00003086-200402000-00021

33. Ishikawa M, Yoshioka K, Urano K, Tanaka Y, Hatanaka T, Nii A. Biocompatibility of cross-linked hyaluronate (Gel-200) for the treatment of knee osteoarthritis. Osteoarthr Cartil. 2014;22(11):1902-1909. doi:10.1016/i.joca.2014.08.00 $\underline{2}$

34. Waddell DD, Joseph B. Delayed Total Knee Replacement with Hylan G-F 20. J Knee Surg. 2016;29(2):159-168. doi:10.1055/s-0034-1395281

35. Kearey P, Popple AE, Warren J, et al. Improvement in condition-specific and generic quality of life outcomes in patients with knee osteoarthritis following single-injection Synvisc: results from the LOBRAS study. Curr Med Res Opin. 2017;33(3):409-419. doi:10.1080/03007995.2016.1260 $\underline{533}$

36. Tammachote N, Kanitnate S, Yakumpor T, Panichkul P. Intra-Articular, Single-Shot Hylan G-F 20 Hyaluronic Acid Injection Compared with Corticosteroid in Knee Osteoarthritis: A DoubleBlind, Randomized Controlled Trial. J Bone Joint Surg Am. 2016;98(11):885-892. doi:10.2106/JBJS.15.00544

37. Dai WL, Lin ZM, Guo DH, Shi ZJ, Wang J. Efficacy and Safety of Hylan versus Hyaluronic Acid in the Treatment of Knee Osteoarthritis. J Knee Surg. 2019;32(3):259-268. doi:10.1055/s-0038-1641142

38. Migliore A, Integlia D, Pompilio G, Di Giuseppe F, Aru C, Brown T. Cost-effectiveness and budget impact analysis of viscosupplementation with hylan G-F 20 for knee and hip osteoarthritis. Clin Outcomes Res. 2019; Volume 11:453-464. doi:10.2147/ceor.s194669

39. Bronstone A, Neary JT, Lambert TH, Dasa V. Supartz (Sodium Hyaluronate) for the Treatment of Knee Osteoarthritis: A Review of Efficacy and Safety. Clin Med Insights Arthritis Musculoskelet Disord. 2019;12. doi:10.1177/1179544119835221

40. Bannuru RR, Brodie CR, Sullivan MC, McAlindon TE. Safety of Repeated Injections of Sodium Hyaluronate (SUPARTZ) for Knee Osteoarthritis: A Systematic Review and Meta-Analysis. Cartilage. 2016;7(4):322-332. doi:10.1177/1947603516642271

41. EUFLEXXA for Osteoarthritis Knee Pain Three Injections Knee Joints. Published 2018. www.euflexx a.com/

42. Bioventus LLC. GELSYN-3. Bioventus OA Knee Pain Relief. Published 2019. www.gelsyn3.com/ 
43. Pavelka K, Uebelhart D. Efficacy evaluation of highly purified intra-articular hyaluronic acid (Sinovial ${ }^{\circledR}$ ) vs hylan G-F20 (Synvisc ${ }^{\circledR}$ ) in the treatment of symptomatic knee osteoarthritis. A double-blind, controlled, randomized, parallel-group non-inferiority study. Osteoarthr Cartil. 2011;19(11):1294-1300. doi:10.1016/j.joca.2011.07.01 $\underline{6}$

44. Leighton R, Fitzpatrick J, Smith H, Crandall D, Flannery CR, Conrozier T. Systematic clinical evidence review of NASHA (Durolane hyaluronic acid) for the treatment of knee osteoarthritis. Open Access Rheumatol Res Rev. 2018;10:43-54. doi:10.2147/OARR R.S162127

45. Fidia Pharma USA Inc. Patients. Hyalgan. Published 2019. hyalgan.com/

46. Miller LE, Sloniewsky MJ, Gibbons TE, Johnston JG, Vosler KD, Nasir S. Long-term clinical benefit and cost-effectiveness of an 8-week multimodal knee osteoarthritis management program incorporating intra-articular sodium hyaluronate (Hyalgan ${ }^{\circledR}$ ) injections. J Pain Res. 2017;10:1045-1054. doi:10.214 7/JPR.S132497

47. Curran MP. Hyaluronic acid (Supartz ${ }^{\circledR}$ ): a review of its use in osteoarthritis of the knee. Drugs Aging. 2010;27(11):925-941. doi:10.2165/11205920-0000000 $\underline{00-00000}$

48. Diraçoğlu D, Tunçay TB, Şahbaz T, Aksoy C. Single versus multiple dose hyaluronic acid: Comparison of the results. J Back Musculoskelet Rehabil. 2016;29(4):881-886. doi:10.3233/BMR-160714

49. Vincent P. Intra-Articular Hyaluronic Acid in the Symptomatic Treatment of Knee Osteoarthritis: A Meta-Analysis of Single-Injection Products. Curr Ther Res - Clin Exp. 2019;90:39-51. doi:10.1016/i.curthere s.2019.02.003

50. McElheny K, Toresdahl B, Ling D, Mages K, Asif I. Comparative Effectiveness of Alternative Dosing Regimens of Hyaluronic Acid Injections for Knee Osteoarthritis: A Systematic Review. Sport Heal A Multidiscip Approach. 2019;11(5):194173811986154. d oi:10.1177/1941738119861545

51. Ha CW, Park YB, Choi CH, et al. Efficacy and safety of single injection of cross-linked sodium hyaluronate vs. three injections of high molecular weight sodium hyaluronate for osteoarthritis of the knee: A double-blind, randomized, multi-center, noninferiority study. BMC Musculoskelet Disord. 2017;18(1):1-10. doi:10.1186/s12891-017-1591-4

52. Richardson C, Plaas A, Block JA. Intra-articular Hyaluronan Therapy for Symptomatic Knee Osteoarthritis. Rheum Dis Clin North Am. Published online 2019. doi:10.1016/i.rdc.2019.04.011
53. Machado RC, Capela S, Rocha FAC. Polysaccharides as viscosupplementation agents: Structural molecular characteristics but not rheology appear crucial to the therapeutic response. Front Med. Published online 2017. doi:10.3389/fmed.2017.00082

54. Han Y, Huang H, Pan J, et al. Meta-analysis Comparing Platelet-Rich Plasma vs Hyaluronic Acid Injection in Patients with Knee Osteoarthritis. Pain Med. 2019;20(7):1418-1429. doi:10.1093/pm/pnz011

55. Li Q, Qi X, Zhang Z. Intra-articular oxygen-ozone versus hyaluronic acid in knee osteoarthritis: A metaanalysis of randomized controlled trials. Int J Surg. 2018;58:3-10. doi:10.1016/j.ijsu.2018.08.007

56. Gregori D, Giacovelli G, Minto C, et al. Association of Pharmacological Treatments with Long-term Pain Control in Patients with Knee Osteoarthritis: A Systematic Review and Meta-analysis. In: JAMA Journal of the American Medical Association. Vol 320. American Medical Association; 2018:2564-2579. doi:1 0.1001/jama.2018.19319

57. Concoff A, Sancheti P, Niazi F, Shaw P, Rosen J. The efficacy of multiple versus single hyaluronic acid injections: A systematic review and meta-analysis. BMC Musculoskelet Disord. 2017;18(1). doi:10.1186/s1 2891-017-1897-2

58. Shen D, Chen M, Chen K, Wang T, Lu L, Yang X. Efficacy of hyaluronic acid after knee arthroscopy: A systematic review and meta-analysis. J Rehabil Med. 2018;50(10):860-865. doi:10.2340/16501977-2366

59. Henrotin Y, Bannuru R, Malaise M, et al.

Hyaluronan derivative HYMOVIS ${ }^{\circledR}$ increases cartilage volume and type ii collagen turnover in osteoarhritic knee: Data from MOKHA study. BMC Musculoskelet Disord. 2019;20(1). doi:10.1186/s12891-019-2667-0

60. Hermans J, Bierma-Zeinstra SMA, Bos PK, Niesten DD, Verhaar JAN, Reijman M. The effectiveness of high molecular weight hyaluronic acid for knee osteoarthritis in patients in the working age: A randomised controlled trial. BMC Musculoskelet Disord. 2019;20(1). doi:10.1186/s12891-019-2546-8

61. Petterson SC, Plancher KD. Single intra-articular injection of lightly cross-linked hyaluronic acid reduces knee pain in symptomatic knee osteoarthritis: a multicenter, double-blind, randomized, placebo-controlled trial. Knee Surgery, Sport Traumatol Arthrosc. 2019;27(6):1992-2002. do $\mathrm{i}: 10.1007 / \mathrm{s} 00167-018-5114-0$

62. Yoon S, Kang JJ, Kim J, Park S, Kim JM. Efficacy and safety of intra-articular injections of hyaluronic acid combined with polydeoxyribonucleotide in the treatment of knee osteoarthritis. Ann Rehabil Med. 2019;43(2):204-214. doi:10.5535/arm.2019.43.2.204 
63. Wang SZ, Wu DY, Chang Q, Guo YD, Wang C, Fan WM. Intra-articular, single-shot co-injection of hyaluronic acid and corticosteroids in knee osteoarthritis: A randomized controlled trial. Exp Ther Med. 2018;16(3):1928-1934. doi:10.3892/etm.20 18.6371
64. Huang Y, Liu X, Xu X, Liu J. Intra-articular injections of platelet-rich plasma, hyaluronic acid or corticosteroids for knee osteoarthritis: A prospective randomized controlled study. Orthopade.

2019;48(3):239-247. doi:10.1007/s00132-018-03659-5 